\title{
A Review on Intelligent Process Automation
}

\author{
Yuvaraja Devarajan \\ Information Technology Professional \\ California, USA
}

\begin{abstract}
Automation - A No-brainer choice for every business leader who want to reduce cost and improve productivity. Companies around the world have been implementing next generation technologies for several years to automate their business operations in order to achieve higher productivity, higher availability, better quality, operating cost reduction etc., As part of this technology evolution, Robotic Process Automation emerged as a game-changing technology which helped enterprises multifold their automation footprint. Due to its versatility, found its way across multiple industry domains automating rule-based repetitive tasks. But with enormous growth of unstructured data \& evolution of new business process trends, demand for cognitive computing tools are on the rise. Intelligent Process Automation / Smart Process Automation, which is a successor of Robotic Process Automation, blends the Robotic Process Automation capabilities with Artificial Intelligence (AI) \& Cognitive computing capabilities. This paper presents a detailed review on Intelligent Process Automation, its evolution, applications and future.
\end{abstract}

\section{Keywords}

Smart Process Automation, SPA, Intelligent Process Automation, IPA, Robotic Process Automation, RPA, Cognitive Automation, Intelligent Automation.

\section{INTRODUCTION}

Robotic Process Automation disrupted the whole automation industry due to its versatile, non-invasive technology that allows bots perform highly repetitive tasks at maximum efficiency \& accuracy. RPA allowed the highly skilled human users to focus on more engaging and challenging activities freeing them from routine, low value repetitive business tasks. With global demand for talented business users on the rise, tremendous growth in unstructured data, evolution of new business processes, organizations across the world are looking for technology solutions that could automate business processes that are non-rule-based harnessing structured / unstructured / semi-structured data.

This search for an advanced RPA tool combined with ever-growing technical advancements in Artificial Intelligence $\&$ Cognitive technologies, paves way for RPA to undergo an inevitable evolution equipping AI and Cognitive capabilities. Intelligent Process Automation / Smart Process Automation integrates the best of Robotic Process Automation, adds Cognitive technologies, Machine Learning and AI allowing organizations to automate non-standard, unstructured, decision making business tasks/processes.

This technology evolution allowed the automation industry to move from typical UI based process automation to real world complex enterprise business process automation. The next section explains in detail about this technology evolution.

\section{EVOLUTION}

Businesses across the world always wanted to improve their productivity \& quality while cutting down operation costs. There were multiple business process automation tools that enabled businesses achieve this goal by automating chunks of business tasks. These tools included screen scraping systems, Macro applications and scripting, workflow automation etc.,

Most of these legacy automation technologies targeted specific application tasks/processes and required humongous amount of work to build, maintain and scale. Most of the times, modifications / technical upgrades in front office / back office applications required these automations to be rebuilt or undergo significant modifications as well. It also increased the overall time and cost required to deploy automation solutions. These task/process-oriented tools solved their purpose, but they didn't provide ability for organizations to analyze and improve their business processes. This demand for improving processes, led these task specific automation tools to adapt Business Process Management principles in order to identify, monitor, analyze, control \& improve the business processes. It also allowed businesses to standardize business processes by continuous evaluation and improvement, reduce costs and improve efficiency. 


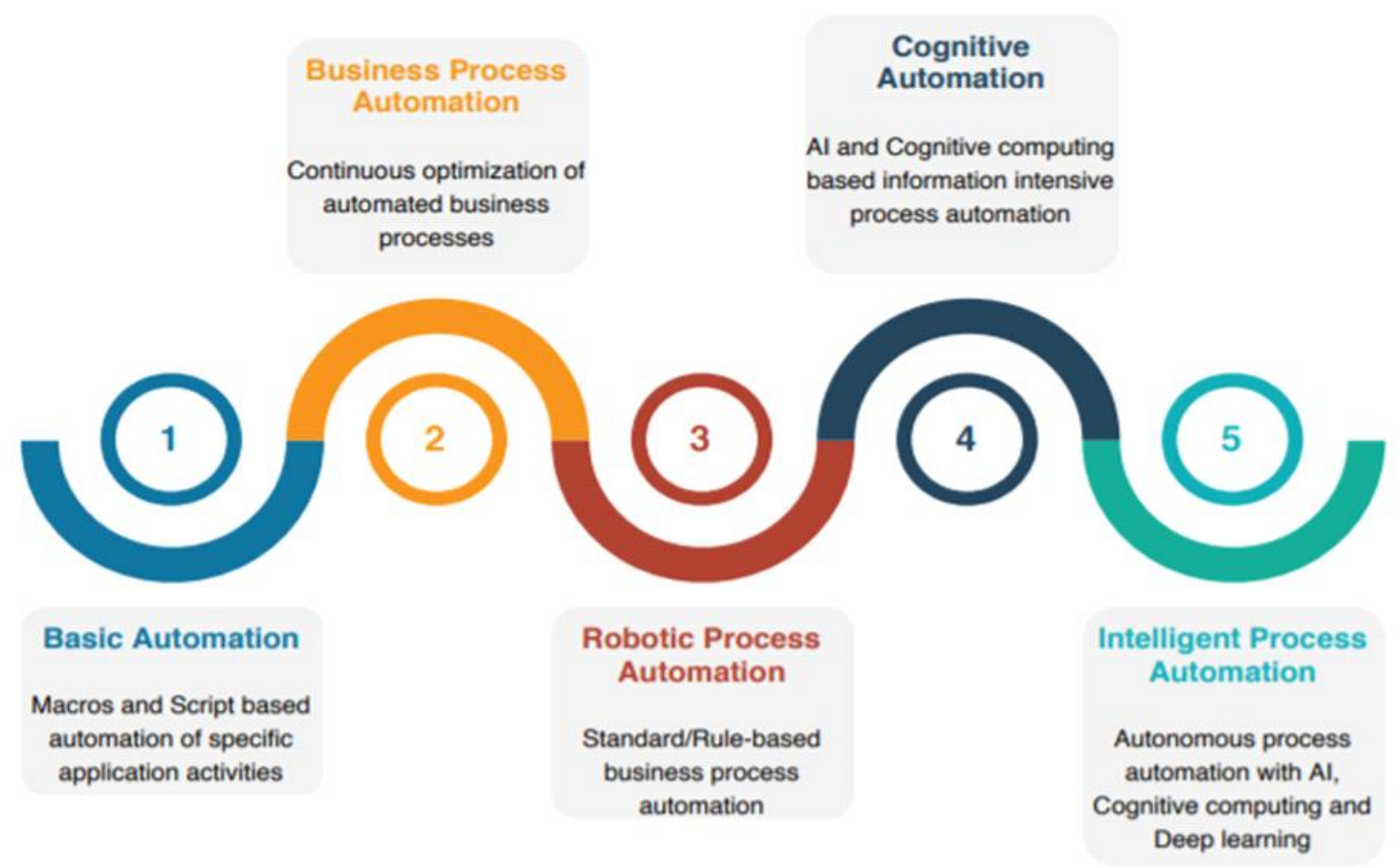

Fig 1: Evolution of Business Process Automation solutions

Riding on the Business process management principles, several new innovative technologies emerged in the automation industry. Robotic Process Automation is one such Next-gen software technology that enabled businesses to automate systematic, repeatable, rule-based business processes. The technology allowed organizations to configure software robots that can emulate human execution via the regular user interfaces. This non-invasive technology disrupted the automation industry as it doesn't require backdoor access to the application, no manual integration efforts with existing applications, also required very minimal/no changes during upgrades/enhancements to business applications. RPA not only performed legacy screen scraping, but also gave flexibility to create smart workflows and ability to work with different back-office applications. With RPA, businesses achieved a magnitude of benefits including Improved accuracy, scalability, productivity, Quality, compliance, reporting , Transparency, employee morale and reduced operating cost.

While RPA made significant improvements in business operations, it still had its own limitations around unstructured data / processes. With ever changing processes and use cases, RPA required continuous configuration / customization of the business rules. On the other hand, Artificial intelligence and Cognitive technologies including Natural Language Processing, Computer Vision, Speech recognition \& Machine Learning had undergone tremendous technological advancements that extend the power of Automation. In order to address and overcome the limitations of RPA, AI and cognitive technologies were adapted and a new wave of automation evolved named Intelligent/Smart Process Automation (IPA/SPA). IPA allowed businesses to automate much broader use cases than RPA, including un-structured processes, the ones that requires user judgement and ones that require collating information from multiple sources to make decision. The Intelligent Process Automation tool set includes a variety of software solutions and explained in detail in subsequent sections.

\section{CONCEPTS}

Intelligent process automation uses a variety of AI tools, Algorithms and technology approaches including Machine Learning / Deep learning, Natural Language Processing, Natural Language Generation, Speech recognition, Computer Vision, Image analytics, Predictive Analytics, Semantic Intelligence etc. allowing automation around Structured, Semi-Structured and Unstructured data. Some of these technology solutions that readily enable business process automation are explained below,

\subsection{Machine Learning (ML)}

Machine learning is a subset of Artificial Intelligence technology that facilitates systems to learn from statistical data and improve from experience without extensive programming. Due to its versality, it could be applied to a variety of problems where significant amount of historic data is available. Machine Learning algorithms essentially build a mathematical model from this historic dataset in order to solve future problems/make predictions and allows systems to improve itself whenever human exceptions are handled. RPA allows businesses to automate processes that are well defined and follow systematic process flow. However, it has limitations around processes that require some form of decision making. Machine learning perfectly fills this gap by learning from the historic decisions made and quickly makes the appropriate decision cutting down the human in loop thereby improving efficiency in processes. 


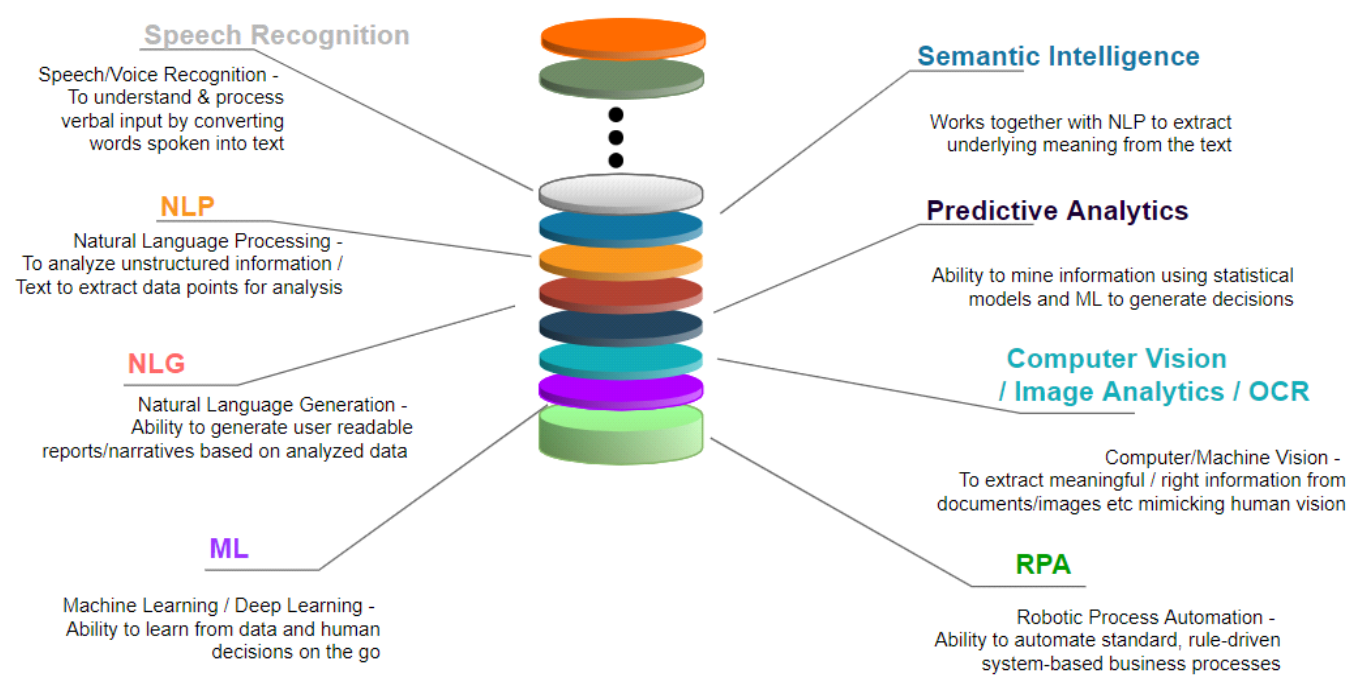

Fig 2: Intelligent Process Automation stack - subset of elements

\subsection{Natural Language Processing - Speech and Text (NLP)}

Natural language Processing (NLP) is also a subset of Artificial Intelligence technology that facilitates systems to understand and process human language. Extracting meaningful information from human language was an extremely complex task in the past, but NLP and Speech recognition enables human-to-machine communication via its powerful algorithms. The NLP text analytics algorithms can also process humongous amount of textual information and extract meaningful information efficiently. While RPA enables automating business processes, it has limitation around processes that require interacting with a human or processing of unstructured text information. NLP \& Text analytics can enable RPA processes to effectively interpreting user request from the text while speech recognition can help interpret user context from speech.

\subsection{Natural Language Generation (NLG)}

Natural Language Generation is also a subset of Artificial Intelligence technology that could manipulate organized/unorganized data into meaningful natural language representation. In simple words, it's a technology that turns system data into human readable narrative. This technology is equally significant since enterprises today require human analysts who would analyze the data and create content. NLG with advanced mining and deep learning capabilities, can analyze historic data sets, identify patterns and generate valuable human readable content. NLG can augment RPA requirements that involve processing data sets to generate content for user.

\subsection{Computer Vision / Image Analytics}

Computer vision, Image analysis, Optical Character recognition are all various techniques that deals with how systems analyze/process/extract information from digital images/files. Most often it's also treated as a subset of Artificial Intelligence with enhanced abilities around pattern recognition and works hand in hand with deep learning object classification. Extracting such qualitative and quantitative data from visual data helps automating processes that require understanding contextual relationships. Computer vision / Image analysis combined with Artificial Intelligence and Deep learning can decipher information from digitized documents and enable better decision making without human intervention.

\subsection{Predictive Analytics}

Predictive analytics is a subset of Machine learning that utilizes statistical algorithms to determine patterns and predict future outcomes based on the existing historical data sets. As with most machine learning techniques, predictive analytics also require large historic datasets to improve the accuracy of its predictions. Looking back at the BPM principles of identifying, monitoring, analyzing and improving business processes, predictive analytics facilitate unlocking significant process insights based on your process flow data. This allows making significant process improvement decisions to multifold efficiency, backed by strong process insight data.

\subsection{Semantic Intelligence}

Semantic Intelligence though sounds like an extension of Natural language processing, its more than just contextual analysis. The application of NLP, AI and Deep learning algorithms enables the text analysis processes to mature as Semantic analysis in order to analyze text, understand the relationship between words and disambiguate their meaning to provide true understanding. While applying RPA to real world scenarios, its critical to not just understand the language but to understand the underlying meaning as well. Semantic Intelligence would be a key asset to decipher the meaning of the text that enables interactive processes more efficient.

\section{APPLICATIONS}

In order to remain competitive in this rapidly evolving technology world, every industry that is going through a digital transformation journey need to make significant investment in disruptive technologies that would maximize their efficiency while keeping the costs low and offer best possible user experience to their customers. 

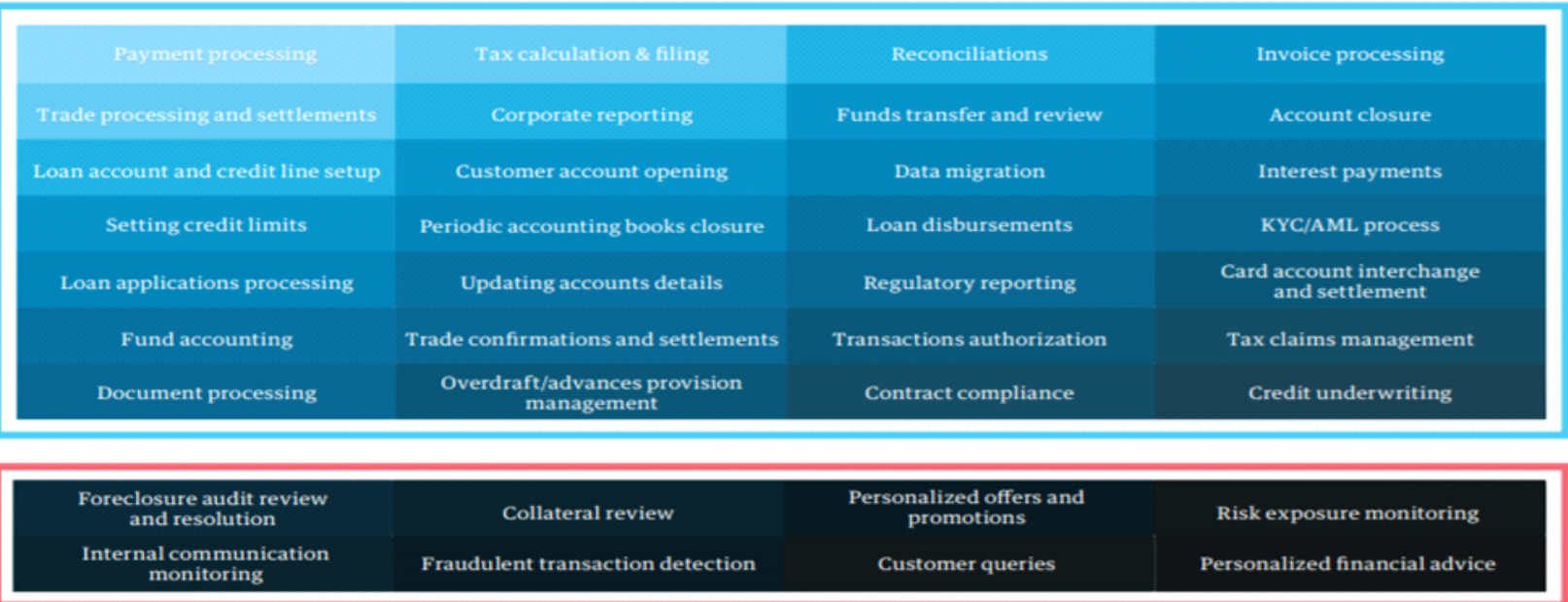

Complex processes

Standardized processes

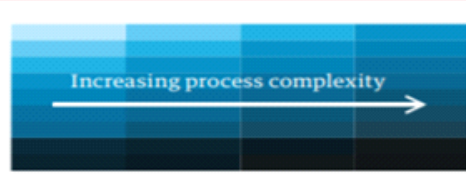

Complex

Fig 3: Automation use cases in Banking/Financial services (Adapted from [9])

Intelligent process automation would be the next step towards achieving that digital transformation powered by digital workforce across a variety of industries including,

- $\quad$ BFSI

- $\quad$ Audit

- $\quad$ Manufacturing

- Retail

- Pharmaceutical

- Aviation

- $\quad$ Oil and Gas

- $\quad$ Energy and resources

- Healthcare

Banking and financial sector deals with tremendous amount of manual business processes that could be automated using Intelligent process automation efficiently. Robotic Process Automation already found its way into BFSI sector, but still couldn't break the back of multiple challenges that exist in that industry. Since it's a heavy customer facing industry, there are numerous use cases that require interacting with the customer, a variety of use cases that allows data flow between multiple interconnected devices including customer mobile apps and several use cases that require analyzing humongous structured, unstructured and semi-structured data sets. Robotic Process Automation wouldn't be able to achieve the level of efficiency and customer satisfaction that the industry demands. In order to make ends meet and orchestrate the flow of all above said processes would require Intelligent process automation powered by Artificial Intelligence, Machine / Deep Learning and variety of Cognitive technologies.

The above snapshot shows an array of use cases across the Banking and Financial services sector ordered by the complexity of the processes. Most of the above use cases were already automated by multiple banks and financial institutions while numerous financial institutions across the world are going through the Process automation journey utilizing Robotic and Intelligent process automation solutions.

In order to stay ahead of competitors, businesses would deploy intelligent process automation solutions in a variety of use cases including,

- $\quad$ Product Recommendation - A very significant use case in Retail, based on customer's preferences and past purchases, AI and ML powered systems can recommend right product quickly

- Chat bots - Intelligent chatbots powered by AI, ML, NLP and Semantic Intelligence would be able to determine customer needs and provide high level of customer engagement

- Portfolio Management - AI driven portfolio management solutions powered by Deep learning, can precisely understand investor requirements \& sentiments to build customized portfolio.

- Access Management - IPA driven automated access management solution would ensure provisioning access to right enterprise resources to the right users in the right context.

- Underwriting - IPA driven automated underwriting solutions powered by AI, ML, NLP can analyze risks quickly and significantly reduce time required to complete underwriting process.

The use case list goes on spanning over multiple business functions and processes.

\section{FUTURE}

In order to boost efficiency, businesses would strive to create digital workplace powered by digital workforce. With rapid development in IoT, we will see a digital conglomeration of Intelligent process automation powered by Artificial Intelligence, Machine Learning, various cognitive technologies, application integrations, robotics, devices, apps etc. It would allow businesses to multifold their efficiency, 
productivity, quality and significantly reduce cost. We will also see tremendous investment flowing towards AI, Deep learning and Neural networking to optimize and improvise business processes.

As Heraclitus quoted, "The only thing that is Constant is Change". We will continue to see enormous changes in the way users and customers experience their digital journey. New set of tools and technologies would evolve that would focus on improving customer / user experience by dynamically analyzing and optimizing business processes as well as communicate these changes to other interconnected devices, processes, apps etc.

\section{CONCLUSION}

Intelligent Process automation allows applying technology to communicate and collaborate with humans and other systems. At this juncture, it's still unsure, if Intelligent Automation would break the back of multiple automation solutions deployed across industries. But it's certain that IPA would allow orchestration of multiple interconnected platforms, processes, devices, systems and functionalities seamlessly. As we observed in the last decade, Artificial Intelligence, Machine Learning and several solutions slowly started working along with humans penetrating through almost every industry. As explained in previous sections, Intelligent process automation will also eventually find its way to work with / without human counter parts across a variety of industries including Manufacturing, BFSI, Audit, Retail, Pharmaceutical etc. Intelligent process automation would in turn drive evolution of new businesses technology trends, strategies, practices and its critical for businesses to adapt \& optimize their business processes to work in coherence with them. As these technology improvements shake up the whole industry, it's crucial for the executives to understand the impact it can bring to the table. It's the moral responsibility of these leaders to educate their human workforce to work along with these technology advancements.

\section{REFERENCES}

[1] ANAGNOSTE, S. (2018) 'The Road to Intelligent Automation in the Energy Sector', Management Dynamics in the Knowledge Economy, 6(3), pp. 489-502. doi: 10.25019/MDKE/6.3.08.

[2] Bellam, S. (2018) 'Robotics vs Machine Learning vs Artificial Intelligence: Identifying the Right Tools for the Right Problems', Credit \& Financial Management Review, 24(2), pp. 1-10. Available at: http://search.ebscohost.com/login.aspx?direct=true \&db= bsh\&AN=131055941\&site=ehost-live
[3] KAUSHIK, S. (2018) 'Critical Parameters for Successful Process Automation', Journal for Quality \& Participation, 41(3), pp. 22-32. Available at: http://search.ebscohost.com/login.aspx?direct=true $\& \mathrm{db}=$ bsh\&AN=132757601\&site=ehost-live

[4] KEDZIORA, D. and KIVIRANTA, H.-M. (2018) 'Digital Business Value Creation with Robotic Process Automation (rpa) in Northern and Central Europe', Management (18544223), 13(2), pp. 161-174. doi: 10.26493/1854-4231.13.161-174.

[5] Woods, Jason M. "Bots, Bid Data, and Auditing the Military." Armed Forces Comptroller 63, no. 3 (Summer 2018): $17-18$

[6] Moffitt, K. C., Rozario, A. M. and Vasarhelyi, M. A. (2018) 'Robotic Process Automation for Auditing', Journal of Emerging Technologies in Accounting, 15(1), pp. 1-10. doi: 10.2308/jeta-10589.

[7] DRISCOLL, TIMOTHY. "Value through Robotic Process Automation." Strategic Finance, no. 3 (March 2018): 70-71.

[8] Papageorgiou, D. (2018) 'Transforming the HR Function Through Robotic Process Automation', Benefits Quarterly, 34, pp. 27-3.

[9] Genpact 2018. Evolution of RPA to IPA available at: https://www.genpact.com/downloadable-content/insight/t he-evolution-from-robotic-process-automation-to-intellig ent-automation.pdf

[10] IBM 2018. Evolution of Process automation available at : https://cdn2.hubspot.net/hubfs/4366404/PowerAI\%20Ca mpaign $\% 202 \% 20$ Content/The $\% 20$ evolution $\% 20$ of $\% 20$ p rocess\%20automation.pdf?t=1533608072577

[11] UiPath 2018. Intelligent process Automation. Available at: https://www.uipath.com/rpa/intelligent-process-automati on

[12] Automation Anywhere 2018. What is Cognitive Automation ? A primer . Available at: https://www.automationanywhere.com/blog/automationas-it-s-best-ally/6099-what-is-cognitive-automation-a-pri mer

[13] Pega Systems 2018. What is the future of Intelligent automation. Available at https://www.pega.com/insights/articles/what-future-intell igent-automation 